\title{
Antioxidant effects of selenocysteine on replicative senescence in human adipose-derived mesenchymal stem cells
}

\author{
Nayoung Suh ${ }^{*} \mathcal{E}$ Eun-bi Lee \\ Department of Pharmaceutical Engineering, Soon Chun Hyang University, Asan 31538, Korea
}

In most clinical applications, human mesenchymal stem cells (hMSCs) are expanded in large scale before their administration. Prolonged culture in vitro results in cellular senescenceassociated phenotypes, including accumulation of reactive oxygen species (ROS) and decreased cell viabilities. Profiling of stem cell-related genes during in vitro expansion revealed that numerous canonical pathways were significantly changed. To determine the effect of selenocysteine (Sec), a rare amino acid found in several antioxidant enzymes, on the replicative senescence in hMSCs, we treated senescent hMSCs with Sec. Supplementation of Sec in the culture medium in late-passage hMSCs reduced ROS levels and improved the survival of hMSCs. In addition, a subset of key antioxidant genes and Sec-containing selenoproteins showed increased mRNA levels after Sec treatment. Furthermore, ROS metabolism and inflammation pathways were predicted to be downregulated. Taken together, our results suggest that Sec has antioxidant effects on the replicative senescence of hMSCs. [BMB Reports 2017; 50(11): 572-577]

\section{INTRODUCTION}

Mesenchymal stem cells (MSCs) are promising sources for development of novel therapeutics due to their abilities for tissue repair and regeneration (1). These cells can be isolated from the stroma of virtually all tissues, including bone marrow, adipose, and umbilical cord (2-4). Numerous animal model experiments have demonstrated that transplantation of ex vivo expanded MSCs have therapeutic effects in various disease settings. Currently, thousands of clinical trials with human MSCs (hMSCs) have been reported for testing their clinical efficacies worldwide (5). To achieve sufficient cell dosage

${ }^{*}$ Corresponding author. Tel: +82-41-530-1628; Fax: +82-41-5303085; E-mail: nysuh@sch.ac.kr

https://doi.org/10.5483/BMBRep.2017.50.11.174

Received 1 September 2017, Revised 14 September 2017, Accepted 18 September 2017

Keywords: Cellular senescence, Human mesenchymal stem cell (hMSC), Reactive oxygen species (ROS), Selenocysteine (Sec) numbers for cell therapy, a reproducible and efficient ex vivo expansion process is required. However, hMSCs undergo a limited number of cell divisions under standard culture conditions, as do all primary human cells (6). This process is known as cellular senescence and is characterized by loss of proliferative capacity, morphological transformation, induction of tumor suppressor networks, increased senescenceassociated $\beta$-galactosidase (SA- $\beta$-gal) activity, and accumulation of reactive oxygen species (ROS) (7). Therefore, it is critical to understand the molecular nature of cellular senescence in hMSCs and to identify intrinsic and/or extrinsic factors affecting this process for clinical applications.

Selenium is an essential nutrient for many organisms, including humans $(8,9)$. At the organismal level, selenium deficiency was associated with various diseases in humans, including Keshan disease, a congestive cardiomyopathy, and Kashin-Beck disease, a chronic, endemic type of osteochondropathy (10). In addition, a selenium concentration decrease in the blood was associated with mortality in elderly human populations (11-13) indicating its role in longevity. At the cellular level, the beneficial effect of selenium on replicative senescence of human fibroblasts has been confirmed (14).

Selenium is thought to function biologically largely through selenoproteins (15). To date, 25 selenoproteins have been identified in humans, and approximately only half of them are known for their functions in redox homeostasis and antioxidant defenses (16). The best-studied selenoproteins are: glutathione peroxidases (Gpx1-Gpx6), which catalyze the glutathione-dependent reduction of organic hydroperoxides and hydrogen peroxide $\left(\mathrm{H}_{2} \mathrm{O}_{2}\right)$; thioredoxin reductases (Txnrd1Txnrd3), which reduce thioredoxins, as well as other substrates; and iodothyronine deiodinase (Dio1-Dio3), which deiodinate thyroid hormones, thereby regulating their activities (17).

All selenoproteins contain selenium in the form of selenocysteine (Sec), a rare amino acid found in both prokaryotes and eukaryotes, in their active sites (18). In contrast to selenium, the beneficial effect of Sec on human health is less clear. Mutations in selenocysteine synthase, which catalyze Sec synthesis, cause neurological disorders in humans (19). However, in Drosophila, selenocysteine synthesis was not essential for oxidative stress and longevity (20). Interestingly, a recent study demonstrated that dietary supplementation of Sec could modulate resistance to environmental stress, with

ISSN: 1976-670X (electronic edition)

Copyright (C) 2017 by the The Korean Society for Biochemistry and Molecular Biology

(ㄷ) This is an open-access article distributed under the terms of the Creative Commons Attribution Non-Commercial License (http://creativecommons.org/licenses/by-nc/4.0) which permits unrestricted non-commercial use, distribution, and reproduction in any medium, provided the original work is properly cited. 
anti-aging effects in Caenorhabditis elegans (C. elegans) (21). Despite these observations at the organismal level, a potential role for Sec in mammalian cells during cellular senescence has not been demonstrated. To test this hypothesis, we demonstrated that supplementation of Sec in the culture medium of late-passage hMSCs significantly decreased ROS levels and improved hMSC survival. Furthermore, when all the selenoproteins were examined, Gpx2, Selenoprotein O (SelO), and SelX showed altered mRNA expression levels after Sec treatment. In addition, genes involved in ROS metabolism including superoxide dismutase 2 (Sod2), NADPH oxidase 1 (Nox1), and Nox4 - also significantly changed their expression with Sec exposure. Extensive bioinformatics analyses revealed that the Sec treatment of senescent hMSCs was predicted to downregulate ROS metabolism and inflammation pathways. Taken together, our results suggest beneficial antioxidant effects of Sec on replicative senescence of hMSCs.

\section{RESULTS AND DISCUSSION}

The hMSC cell lines were established from three donors and expanded in vitro to determine the senescence-associated phenotypes. A long-term growth curve revealed that earlypassage cells maintained high proliferation rates until they reached senescence, with $35 \pm 3.69$ cumulative population doublings (CPDs) (Fig. 1A). Compared to early-passage hMSCs, late-passage cells showed characteristic flattened-cell morphologies (Fig. 1B). The number of viable cells was then determined by a MTS [3-(4,5-dimethylthiazol-2-ul)-5-(3-carboxymethoxyphenyl)-2-(4-sulfophenyl)-2H-tetrazolium] assay. Consistent with results from long-term growth experiments, the MTS assay showed more than a $60 \%$ reduction of proliferation capacities of late-passage cells (Fig. 1C). It has been well known that oxidative stress and ROS production are major causes of cellular senescence and organismal aging (22). Using


Fig. 1. Characterization of senescence-associated changes in ex vivo expanded-hMSCs. (A) hMSCs were isolated from the adipose tissue of three donors and evaluated for the cumulative population doublings (CPD). (B) Representative images of hMSCs at early and late passage. Scale bar: $100 \mu \mathrm{m}$. (C) Viable cells were examined by MTS assay in young (passage 3-6) and senescent (passage 20-26) hMSCs. Error bars denote the standard error of the mean of 3 experiments $(* \mathrm{P}<0.0001)$. (D) $\mathrm{H}_{2} \mathrm{O}_{2}$ levels produced in young and senescent cells were quantified by the Amplex ${ }^{\mathbb{R}}$ Red assay. Error bars denote the standard error of the mean of 3 experiments $(* P<0.0001)$.
Amplex ${ }^{R}$ Red reagents, which reacts with $\mathrm{H}_{2} \mathrm{O}_{2}$ to produce a fluorescent oxidation derivative in the presence of peroxidase, we observed a 2.2-fold increase in $\mathrm{H}_{2} \mathrm{O}_{2}$ in senescent cells (Fig. 1D). Based on the observed senescence phenotypes, we categorized hMSCs into two groups (early [E, passage 3-6] and late [L, passage 20-26]) and performed further analyses.

To determine the molecular regulation of cellular aging in hMSCs, we performed a PCR array with early- and late-passage hMSCs. The expression levels of 84 genes related to identification, growth, and differentiation of stem cells were examined. Consistent with previous reports on the mRNA expression profiles of bone marrow-derived MSCs (23), early- and latepassage hMSCs showed extensive expression profile changes in stem cell-related genes. Compared with early-passage hMSCs, 7 genes were upregulated, and 20 genes were downregulated more than 2-fold in late-passage hMSCs (Fig. 2A, black and gray dots respectively) (Fig. 2A). Interestingly, stem cell specific markers, such as cell cycle regulators (Ccna2,
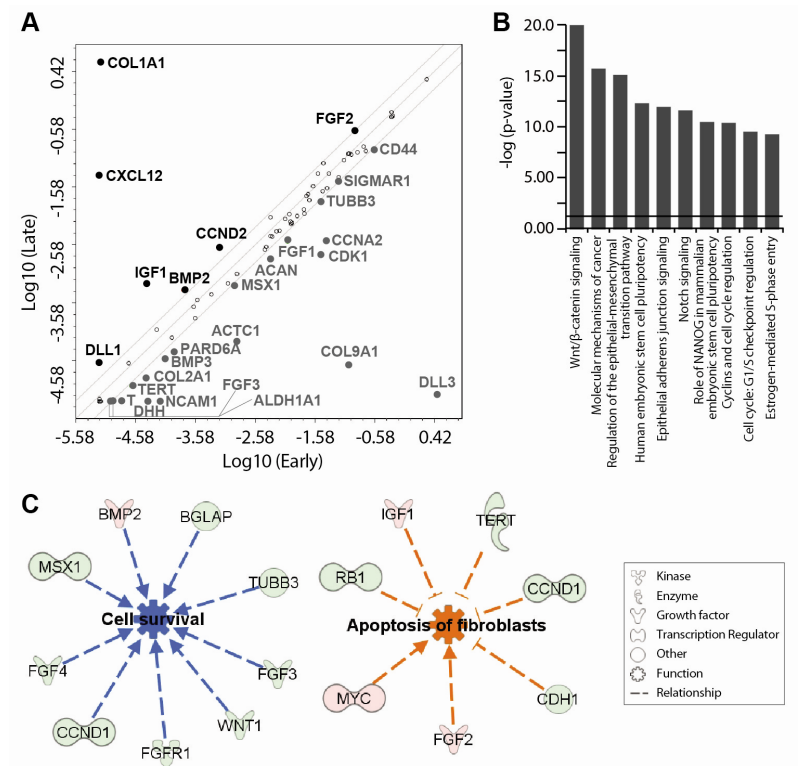

Fig. 2. Gene expression profiles of hMSCs during in vitro aging. (A) The scatter plot of young and senescent hADSCs using $\mathrm{RT}^{2}$ Profiler $^{\mathrm{TM}}$ PCR Array Human Stem Cell, which contains 84 genes related to identification, growth, and differentiation of stem cells. Genes up-regulated or down-regulated in late-passage hMSCs by at least 2-fold are indicated with black and gray dots, respectively. (B) Regulated top 10 canonical pathways significantly altered by ex vivo amplification of hMSCs were shown by using Ingenuity Pathways Analysis (IPA) (Benjamini-Hochberg multiple test-corrected P-values $<0.05$ ). (C) Analysis of the expression profile data with the cellular functions by using IPA software. The molecule types and relationships were indicated in a boxed legend. The colors in the molecular types reflect the expression levels in the dataset. The relationships were presented in blue and orange dashed lines representing the inhibition and activation of the cellular functions, respectively. 
Cdk1, Fgf1, Fgf3, Pard6a), genes regulating symmetric/ asymmetric cell division (Dhh, Pard6a) and cell adhesion molecules (CD44, Col9a1, Ncam1), were significantly downregulated. In addition, stem cell differentiation markers, including embryonic cell lineage markers (Actc1, Msx1), mesenchymal cell lineage markers (Acan, Col2a1, Col9a1), and neural cell lineage markers (CD44, Ncam1, Sigmar1, Tubb3), were also greatly downregulated in senescent cells. However, cytokines and growth factors were both upregulated (Bmp2, Cxcl12, Fgf2, Igf1) and downregulated (Bmp3, Fgf1, Fgf3), implying a dramatic change in secreted molecules.

We next evaluated canonical pathways associated with in vitro aging of hMSCs using Ingenuity Pathway Analysis (IPA) software. Function and network analysis revealed predominant enrichment of 118 canonical pathways (Benjamini-Hochberg multiple test-corrected P-values $<0.05$ ) including those of Wnt/B-catenin, Notch, and cancer signaling (Fig. 2B). To better understand the underlying mechanisms for senescence phenotypes, we focused on the changes in cellular functions, which were predicted based on the expression profile data. Notably, in late-passage hMSCs, significant changes were observed in several important cellular functions such as the inhibition of cell survival and the activation of apoptosis in fibroblasts (Fig. 2C). Taken together and consistent with previous reports, senescent hMSCs exhibited systematic deregulation of stem cell properties, including proliferation/ differentiation capabilities, secretion of trophic factors, and associated signaling pathways.

It has been reported that serum levels of micronutrient selenium are closely related to human mortality $(12,13)$. At the cellular level, selenium levels can extend the replicative life span of human fibroblasts when added during early-stage expansion (14). In addition, dietary supplementation of Sec, a selenium-containing cysteine, leads to lifespan extension in $C$. elegans (21). However, the relationship between Sec and replicative senescence in hMSCs is not known. To determine the effect of Sec during replicative senescence, we treated senescent hMSCs with two different concentrations of Sec. The proliferation rate of hMSCs was measured by MTS assay. Sec supplementation increased the proliferation capability to $28.7 \%$ ( $50 \mathrm{nM} \mathrm{Sec)}$ and $36.9 \%$ (100 nM Sec) compared to the untreated senescent hMSCs (Fig. 3A). Next, we monitored the $\mathrm{H}_{2} \mathrm{O}_{2}$ status after $\mathrm{Sec}$ treatment. Compared with untreated late-passage $\mathrm{hMSCs}, \mathrm{H}_{2} \mathrm{O}_{2}$ levels were significantly reduced to $14.6 \%$ and $22.4 \%$ with $50 \mathrm{nM}$ and $100 \mathrm{nM} \mathrm{Sec}$, respectively (Fig. 3B). Moreover, late-passage hMSCs grown in $100 \mathrm{nM} \mathrm{Sec}$ for 24 hours showed more discrete cell morphologies compared to the flattened morphologies seen in senescent cells (Fig. 3C). In conclusion, Sec supplementation exerts positive effects on proliferation capability of senescent hMSCs in part by reducing $\mathrm{H}_{2} \mathrm{O}_{2}$ accumulation.

To gain further insight into the underlying mechanisms of Sec treatment on senescent hMSCs, we analyzed the expression levels of all 25 human selenoproteins using
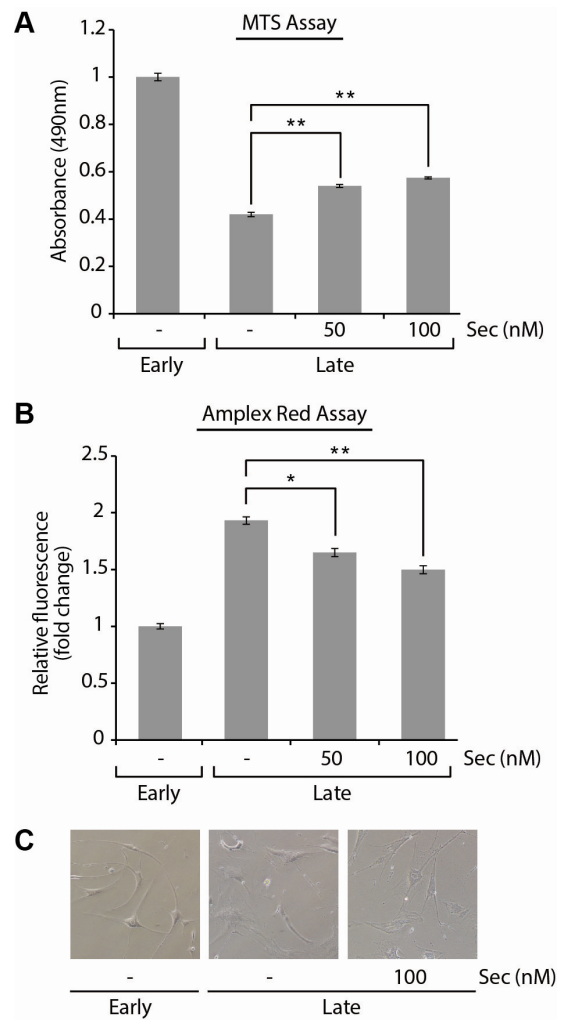

Fig. 3. Effects of $\mathrm{Sec}$ on survival and $\mathrm{H}_{2} \mathrm{O}_{2}$ accumulation in senescent hMSCs. (A) Senescent hMSCs were cultured with 0, 50, and $100 \mathrm{nM}$ of Sec for 24 hours and then cell growth rate was measured using MTS assay. Error bars denote standard error of the mean of 6 experiments (**P $<0.0001)$. (B) $\mathrm{H}_{2} \mathrm{O}_{2}$ production was quantified by Amplex ${ }^{\mathbb{R}}$ Red on early, late, and late-passage hMSCs treated with $50 \mathrm{nM}$ and $100 \mathrm{nM}$ Sec for 24 hours. Error bars denote the standard error of the mean of 6 experiments (*P $=0.001 ; * * \mathrm{P}<0.0001)(\mathrm{C})$ Representative photographs of hMSCs on young and senescent cells with or without $100 \mathrm{nM}$ selenocysteine treatment.

qRT-PCR analysis (Fig. 4A). The other 24 selenoproteins were all expressed in hMSCs, except for Dio1 (which was not detectable in this condition). We observed that in response to Sec treatment, Gpx2 was dramatically up-regulated (32.9X relative to the untreated late-passage hMSCs, $\mathrm{P}<0.001)$, whereas SelO (0.76X, P < 0.05) and SelX $(0.77 X, \mathrm{P}<0.05)$ were statistically down-regulated. Since selenium supplementation can modulate selenoproteins at both mRNA and protein levels (14), we cannot rule out the possibility of translational control after Sec treatment. We next monitored the expression of genes involved in ROS metabolism, including superoxide dismutases (Sod 1-3) and NADPH oxidases (Nox1 and 4), after Sec treatment (Fig. 4B). A mitochondrial protein, Sod2 (2.1X compared to the untreated late-passage hMSCs, $\mathrm{P}<0.01$ ) and Nox1 (22.3X, P $<0.001)$ were significantly up-regulated and Nox4 (0.35X, P < 0.05) was down-regulated. 
A

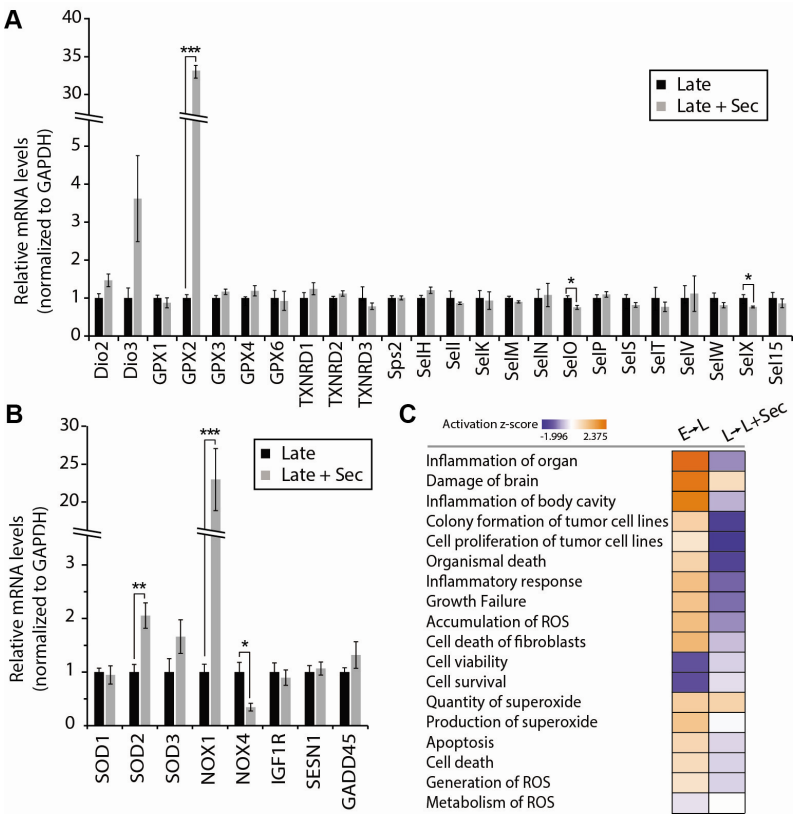

Fig. 4. Regulation of selenoproteins and genes involved in ROS metabolism in response to Sec supplementation in senescent hMSCs. (A) mRNA levels of selenoproteins were evaluated by qRT-PCR analysis. Error bars denote the standard error of the mean of four experiments (*P $<0.05 ; * * * P<0.001)$. (B) Expression levels of genes associated with ROS metabolism were measured by qRTPCR analysis upon Sec treatment. Error bars denote standard error of the mean of four experiments (*P $<0.05 ; * * P<0.01$; $* * * P<0.001)$. (C) The heat map of a comparison analysis on disease and function was generated using IPA software. Pathways were sorted by activation Z-scores, which were calculated by total - $\log ($ P-value $)$ from Fisher's Exact test across compared observations (observation 1, early to late transition; observation 2, late to late with Sec treatment transition).

Using mRNA expression data on both selenoproteins and other genes involved in ROS metabolism, we next performed a comprehensive bioinformatic analysis using IPA software. Specifically, we compared the diseases and functions of early to late-passage and late to late-passage cells with Sec treatment (Fig. 4C). As expected, during ex vivo expansion of hMSCs, inflammation, ROS metabolism, cell death, and apoptotic pathways were all increased (Fig. 4C, left column, red) in senescent cells. In contrast, cell viability and survival functions were greatly decreased in senescent cells (Fig. 4C, left column, blue). However, there were striking changes in those pathways when cells were exposed to the Sec (Fig. 4C, right column). Indeed, Sec supplementation on senescent hMSCs was predicted to decrease ROS metabolism pathways, such as ROS accumulation, superoxide quantity, superoxide production, and generation and metabolism of ROS (Fig. 4C, right column, activation z-score from -0.8825 to 0.038964$)$. Furthermore, cell viability and survival pathways were predicted to increase upon Sec treatment (activation z-score: -0.40095 and
-0.30488). Interestingly, inflammation-related biofunctions, including inflammation of organ and inflammatory response, were anticipated to decrease with $\mathrm{Sec}$ supplementation (activation z-score: -0.8849 and -1.13137 ). Considering the anti-inflammatory properties of selenium in human health (24), this was not a surprising finding.

In conclusion, our findings show the beneficial effects of Sec during hMSC senescence for the first time. We observed a significant increase in cell viability and a selective regulation of selenoproteins and genes involved in ROS metabolism upon Sec treatment on senescent hMSCs. Comparison analysis on diseases and functions further provide evidence for the antioxidant response of Sec on cellular senescence in hMSCs.

\section{MATERIALS AND METHODS}

\section{Isolation and culture conditions of hMSCs}

This study was carried out according to the guidelines of the Institutional Review Board of Soon Chun Hyang University. hMSCs were isolated from human adipose tissue of three donors (age range of 42-51 years) and cultured as previously described (25-28). The hMSCs obtained were cultured in DMEM with $10 \%$ FBS, 100 units/ml penicillin and $100 \mu \mathrm{g} / \mathrm{ml}$ streptomycin (Gibco, Gaithersburg, MD, USA) in a humidified atmosphere containing $5 \% \quad \mathrm{CO}_{2}$ at $37^{\circ} \mathrm{C}$. Medium was replaced every 3 days and the cells were used at passage 3 to 6 for early passages and passage 20 to 26 for late passages. For $\mathrm{Sec}$ treatment, late-passage hMSCs were incubated with different concentrations $(0,50$, or $100 \mathrm{nM}$ ) of Sec (SigmaAldrich, St. Louis, MO, USA) for 24 hours.

\section{Cell proliferation assay}

Cell proliferation was measured using a colorimetric assay with 3-(4,5-dimethylthiazol-2-ul)-5-(3-carboxymethoxyphenyl)2-(4-sulfophenyl)-2H-tetrazolium (MTS) (Promega, Madison, WI, USA) (29). Briefly, cells were plated $3 \times 10^{3}$ cells per well in 96-well plate on day 1 and then treated with different concentrations $(0,50$, or $100 \mathrm{nM})$ of Sec. on day 2. After 24 hours of incubation, $20 \mu \mathrm{l}$ of MTS solution was added to each well and incubated for 4 hours at $37^{\circ} \mathrm{C}$. The absorbance was then recorded at $490 \mathrm{~nm}$ using a BioTek Epoch2 microplate reader (BioTek, Winooski, VT, USA). Each assay was performed in three replicate wells for each condition.

\section{Hydrogen peroxide $\left(\mathrm{H}_{2} \mathrm{O}_{2}\right)$ measurement}

For the quantitative measurement of $\mathrm{H}_{2} \mathrm{O}_{2}$ levels, an Amplex ${ }^{\circledR}$ Red Hydrogen Peroxide/Peroxidase Assay Kit (ThermoFisher Scientific, Waltham, MA, USA) was used in accordance with the manufacturer's instructions. Briefly, hMSCs were plated 6 $\times 10^{4}$ cells per well in a 6-well plate, and senescent hMSCs were incubated with different concentration $(0,50$, or $100 \mathrm{nM})$ of Sec. After 24 hours, cells were detached with $0.25 \%$ trypsin/EDTA and suspended in Krebs-Ringer phosphate (KRPG) buffer (145 mM NaCl, $5.7 \mathrm{mM}$ sodium phosphate, 
$4.86 \mathrm{mM} \mathrm{KCl}, 0.54 \mathrm{mM} \mathrm{CaCl} 2,1.22 \mathrm{mM} \mathrm{MgSO}_{4}, 5.5 \mathrm{mM}$ glucose, $\mathrm{pH}$ 7.35). The reaction mixture $\left(50 \mu \mathrm{M}\right.$ Amplex ${ }^{\mathbb{R}}$ Red reagent and $0.1 \mathrm{U} / \mathrm{ml} \mathrm{HRP} \mathrm{in} \mathrm{KRPG} \mathrm{buffer)} \mathrm{was} \mathrm{pre-warmed} \mathrm{at}$ $37^{\circ} \mathrm{C}$ for 10 minutes and then incubated with $1 \times 10^{4}$ cells in 96-well plate. After incubation for 90 minutes, fluorescence was measured at $540 \mathrm{~nm}$ excitation and $590 \mathrm{~nm}$ emission using BioTek Epoch2 microplate reader.

\section{Quantitative real-time PCR (qRT-PCR)}

Total RNA was isolated from cultured cells using Trizol (ThermoFisher Scientific, Waltham, MA, USA) according to the manufacturer's protocol. For quantitative real-time PCR (qRT-PCR) analysis of mRNA, 300 ng of total RNAs were transcribed with random hexamers and SuperScript IV reverse transcriptase (ThermoFisher Scientific, Waltham, MA, USA). The qRT-PCR assay was then performed using a Power SYBR Green PCR master mix on a QuantStudio 6 Flex Real-Time PCR system (ThermoFisher Scientific, Waltham, MA, USA). The primers used in the qRT-PCR can be seen in Supplementary Table 1. Glyceraldehyde 3-phosphate dehydrogenase $(G A P D H)$ was used as an endogenous normalization control.

\section{PCR array and network analysis}

The RT ${ }^{2}$ Profiler $^{\text {TM }}$ PCR Array Human Stem Cell (PAHS-405Z) (Qiagen, Hilden, Germany) was used as previously described $(25,30)$. Briefly, $400 \mathrm{ng}$ of total RNAs were treated with DNase I and then reverse transcribed using the $\mathrm{RT}^{2}$ First Strand Kit (Qiagen, Hilden, Germany). PCR quantification was done on a 7900 Real-Time PCR system using RT ${ }^{2}$ SYBR Green qPCR Master Mixes. Data analysis was performed using online analysis software (http://pcrdataanalysis.sabiosciences.com/pcr/ arrayanalysis.php) (Qiagen, Hilden, Germany). The canonical pathways, functional network, and comparison analysis were performed using Ingenuity Pathway Analysis (IPA) software (Qiagen, Hilden, Germany).

\section{ACKNOWLEDGEMENTS}

This work was supported by the Soon Chun Hyang University Research Fund (No. 20160225), and the Basic Science Research Program through the National Research Foundation of Korea (NRF) from the Ministry of Education, Science, and Technology (2016R1D1A1B03935929).

\section{CONFLICTS OF INTEREST}

The authors have no conflicting interests.

\section{REFERENCES}

1. Bianco P, Cao X, Frenette PS et al (2013) The meaning, the sense and the significance: translating the science of mesenchymal stem cells into medicine. Nat Med 19, 35-42
2. da Silva Meirelles L, Chagastelles PC and Nardi NB (2006) Mesenchymal stem cells reside in virtually all post-natal organs and tissues. J Cell Sci 119, 2204-2213

3. Brighton CT and Hunt RM (1991) Early histological and ultrastructural changes in medullary fracture callus. J Bone Joint Surg Am 73, 832-847

4. Brighton CT and Hunt RM (1997) Early histologic and ultrastructural changes in microvessels of periosteal callus. J Orthop Trauma 11, 244-253

5. Daley GQ (2012) The promise and perils of stem cell therapeutics. Cell Stem Cell 10, 740-749

6. Hayflick L and Moorhead PS (1961) The serial cultivation of human diploid cell strains. Exp Cell Res 25, 585-621

7. Kuilman T, Michaloglou C, Mooi WJ and Peeper DS (2010) The essence of senescence. Genes Dev 24, 2463-2479

8. Choi YJ, Yang SG, Jung MM, Kim BS, Yun SG, Choi CY (2015) Effects of waterborne selenium on toxic and physiological stress response in goldfish, Carassius auratus. Mol Cell Toxicol 11, 35-46

9. Hatfield DL, Tsuji PA, Carlson BA and Gladyshev VN (2014) Selenium and selenocysteine: roles in cancer, health, and development. Trends Biochem Sci 39, $112-120$

10. Combs GF and Combs SB (1986) The role of selenium in nutrition, Academic Press, Orlando 327-399

11. Olivieri O, Stanzial AM, Girelli D et al (1994) Selenium status, fatty acids, vitamins $A$ and $E$, and aging: the Nove Study. Am J Clin Nutr 60, 510-517

12. Akbaraly NT, Arnaud J, Hininger-Favier I, Gourlet V, Roussel AM and Berr C (2005) Selenium and mortality in the elderly: results from the EVA study. Clin Chem 51, 2117-2123

13. Ray AL, Semba RD, Walston J et al (2006) Low serum selenium and total carotenoids predict mortality among older women living in the community: the women's health and aging studies. J Nutr 136, 172-176

14. Legrain Y, Touat-Hamici Z and Chavatte L (2014) Interplay between selenium levels, selenoprotein expression, and replicative senescence in WI-38 human fibroblasts. J Biol Chem 289, 6299-6310

15. Bellinger FP, Raman AV, Reeves MA and Berry MJ (2009) Regulation and function of selenoproteins in human disease. Biochem J 422, 11-22

16. Kryukov GV, Castellano S, Novoselov SV et al (2003) Characterization of mammalian selenoproteomes. Science 300, 1439-1443

17. Reeves MA and Hoffmann PR (2009) The human selenoproteome: recent insights into functions and regulation. Cell Mol Life Sci 66, 2457-2478

18. Li F, Lutz PB, Pepelyayeva Y, Arner ES, Bayse CA and Rozovsky S (2014) Redox active motifs in selenoproteins. Proc Natl Acad Sci U S A 111, 6976-6981

19. Puppala AK, French RL, Matthies D, Baxa U, Subramaniam S and Simonovic M (2016) Structural basis for early-onset neurological disorders caused by mutations in human selenocysteine synthase. Sci Rep 6, 32563

20. Hirosawa-Takamori M, Chung HR and Jackle H (2004) Conserved selenoprotein synthesis is not critical for oxidative stress defence and the lifespan of Drosophila. 
EMBO Rep 5, 317-322

21. Kim JS, Kim SH and Park SK (2017) Selenocysteine modulates resistance to environmental stress and confers anti-aging effects in C. elegans. Clinics (Sao Paulo) 72, 491-498

22. Stolzing A and Scutt A (2006) Age-related impairment of mesenchymal progenitor cell function. Aging Cell 5, 213-224

23. Wagner W, Horn P, Castoldi M et al (2008) Replicative senescence of mesenchymal stem cells: a continuous and organized process. PLoS One 3, e2213

24. Rayman MP (2000) The importance of selenium to human health. Lancet 356, 233-241

25. Park SK, Lee JS, Choi EK, You D, Kim CS and Suh N (2014) Global knockdown of microRNAs affects the expression of growth factors and cytokines in human adipose-derived mesenchymal stem cells. BMB Rep 47, 469-474
26. Zuk PA, Zhu M, Mizuno H et al (2001) Multilineage cells from human adipose tissue: implications for cell-based therapies. Tissue Eng 7, 211-228

27. Lee JS, Song DY, Cho WG et al (2015) Transplantation of human mesenchymal stem cells into the cisterna magna and its neuroprotective effects in a parkinsonian animal model. Mol Cell Toxicol 11, 373-385

28. Kim PH, Na SS, Lee B, Kim JH, Cho JY (2015) Stanniocalcin 2 enhances mesenchymal stem cell survival by suppressing oxidative stress. BMB Rep 48, 702-707

29. Lee JH, Kim IW, Kim SH, Yun EY, Nam SH (2015) Anticancer activity of CopA3 dimer peptide in human gastric cancer cells. BMB Rep 48, 324-329

30. Cha HJ, Ahn JI, Jeong JY et al (2015) Identification of modulated mRNAs and proteins in human primary hepatocytes treated with non-steroidal anti-inflammatory drugs. Mol Cell Toxicol 11, 335-342 